\title{
Research on Influence of Road Tunnels with Different Lanes on Surrounding Rock Characteristic Curve
}

\author{
SunYang ${ }^{1}$, ZhangChengke ${ }^{1}$, YuJunping ${ }^{1 *}$, WuJiangpeng ${ }^{1}$, ZhuLiqing ${ }^{1}$ \\ ${ }^{1}$ Jiangxi Transportation Research Institute, Nanchang 330200
}

\begin{abstract}
Convergence confinement method is an important guidance method for tunnel construction and support design. Numerical simulation method was used to comparatively analyze the ground reaction curve and the plastic zone under different rock grade and roadway tunnel size. The results show that the change of tunnel size has different effects on the maximum deformation of the tunnel arch crown, the ground reaction curve and the plastic zone range. Finally, some suggestions were put forward for the construction and optimization of the large span arch tunnel support structure. The research results may provide some guidance for related engineering
\end{abstract}

\section{Introduction}

In this paper, there are various deficiencies in the analytical solutions in actual highway tunnel engineering, according to the monitoring data, the mechanical properties of the surrounding rock of the tunnel are obtained by inversion ${ }^{[1] \sim[3],}$ apply numerical simulation analysis method, based on the design needs of the secondary lining support of the tunnel, consider two different tunnel sizes (different lanes), study its different surrounding rock characteristic curves ${ }^{[4] \sim[5]}$ and surrounding rock plastic zone morphology respectively, and conduct comparative analysis.

\section{Project Overview}

A highway tunnel entrance pile number $\mathrm{K} 7+595$, the exit pile number is $\mathrm{K} 8+970$, the length of the tunnel is $1375 \mathrm{~m}$. Tunnel construction boundary: clear width $16 \mathrm{~m}$, carriageway width $3 \times 3.75 \mathrm{~m}$, carriageway height $5 \mathrm{~m}$. The ground elevation of the tunnel crossing section is $55 \sim 230 \mathrm{~m}$, the terrain is undulating and hilly. The tunnel cover is Quaternary residual soil, slope alluvial gravel soil, gravel-containing silty clay, the underlying fully weathered $\sim$ slightly weathered tuff of the Late Jurassic Xishantou Formation, joint faults are developed, mainly distributed at the bottom of the coastal plain area and the surface of the low mountain and hilly area. A total of 6 secondary faults and dense joints have been discovered in the tunnel site area that have a certain impact on tunnel excavation. affected by regional faults and secondary faults, the joints and cracks in the tunnel site are relatively developed ${ }^{[6]}$. The surrounding rock of the tunnel is divided into three types of surrounding rock of III, IV, and V according to stratum lithology, geological structure, weathering characteristics and hydrogeological conditions $^{[7]}$.

\section{Numerical simulation analysis plan}

The analysis section is selected on the right side of the tunnel, the specific pile number is YK $7+671.00$, passing through the SZ4 borehole, and the surrounding rock of grade IV and $\mathrm{V}$ are considered respectively. The calculation model and the finite element analysis model are shown in Figure 1 and Figure 2( Taking into account the symmetry of the problem, the model only chooses half of the symmetry. The model diagram shows three lanes; if it is set to a two lane tunnel, the excavation size will become: height: $11.287 \mathrm{~m}$, half span: $7.47 \mathrm{~m}$, and the boundary size remains unchanged).

\begin{tabular}{cccccc} 
Table 1 & \multicolumn{4}{c}{ Calculation parameters of elasto-plastic model } \\
\hline $\begin{array}{c}\text { Surrounding } \\
\text { rock level }\end{array}$ & $\begin{array}{c}E \\
\mathrm{GPa}\end{array}$ & $\mu$ & $\begin{array}{c}c \\
\mathrm{kPa}\end{array}$ & $\begin{array}{c}{ }^{\circ} \\
\mathrm{kN} \cdot \mathrm{m}^{-3}\end{array}$ \\
\hline IV & 1.122 & 0.25 & 513.7 & 44.17 & 24.5 \\
V & 0.41 & 0.384 & 208.2 & 31.32 & 21.0
\end{tabular}

\footnotetext{
* Corresponding author:2574544207@qq.com
} 


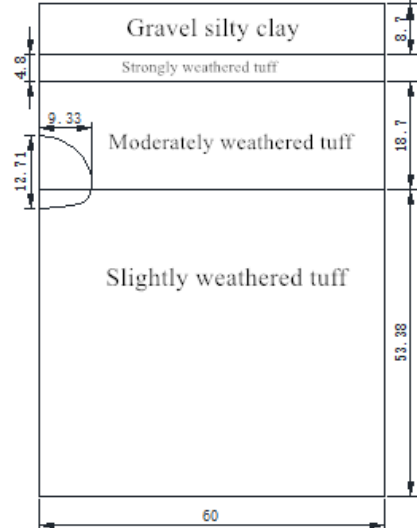

Fig. 1 Calculation model

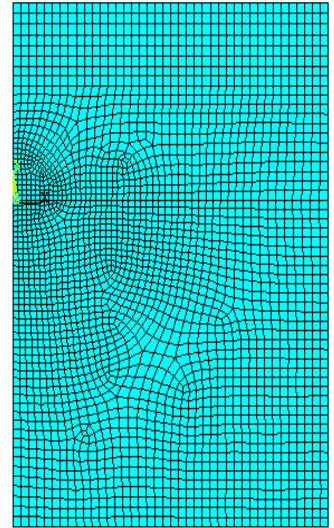

Fig. 2 FEM analysis model

\section{Analysis of Tunnel Numerical Simulation Results}

\subsection{Three-lane Class IV surrounding rock tunnel}

Figure 3 shows the characteristic curve of surrounding rock at the top of the arch. In the figure, $\mathrm{Pi}$ is the force on the cave wall, P0 is the initial ground stress, and $\mathrm{u}$ is the displacement of the top of the arch around the cave.

It can be seen from Figure 3 that the characteristic curve of the surrounding rock at the top of the arch is composed of three sections: a straight line, a descending curve and an ascending curve, that is, with the excavation of the tunnel, the surrounding rock around the tunnel gradually develops from elastic to elasto-plastic state, The plastic zone continues to expand, the elastoplastic boundary is about $90 \%$ of the initial ground stress release, and the top arch is deformed to $10 \mathrm{~mm}$ after the excavation is completed. Figure 4 shows the distribution of plastic zone in surrounding rock after tunnel excavation is completed, in the figure, the plastic zone of the surrounding rock is more evenly distributed around the cave, and the depth of the plastic zone at the top arch is the largest, reaching nearly $1.4 \mathrm{~m}$.

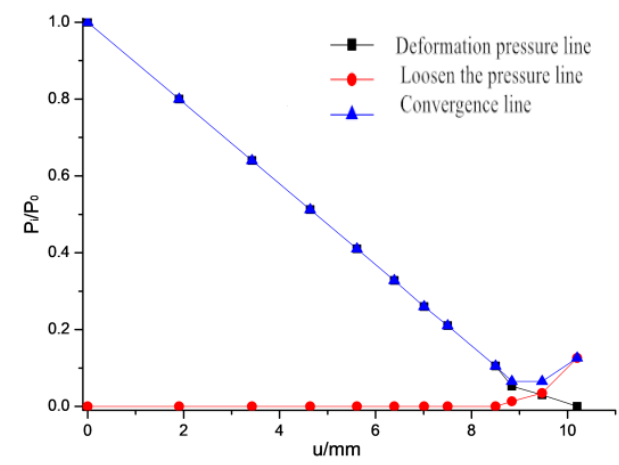

Fig. 3 GRC of three lane tunnel for arch crown

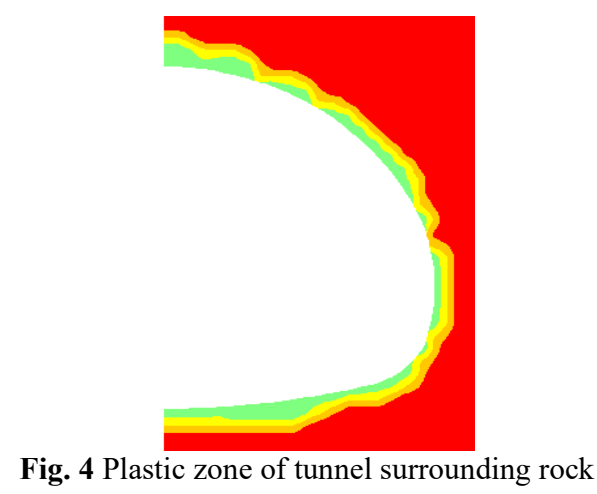

\subsection{Three-lane V-level surrounding rock tunnel}

Compared with Grade IV surrounding rock, the shape of the convergence characteristic curve of surrounding rock is not much different, the displacement value of the tunnel wall increases significantly with the deterioration of the surrounding rock, the lowest point of the arch top position convergence characteristic curve occurs when the dome settlement value is about $26 \mathrm{~mm}$, the corresponding surrounding rock pressure is about $20 \%$ of the initial ground stress value, under this stress release, the top arch part begins to appear plastic zone.

Combined with the development process of the plastic zone with stress release in Figure 6, it can be seen, the rock plastic zone around the cave first occurs and expands at the side wall, then gradually developed to the arch and bottom, after the excavation is completed, the depth of the plastic zone at the top arch reaches $4 \mathrm{~m}$, which is the largest plastic zone depth around the cave. This conforms to the regularity of the development of plastic zone of surrounding rock when the lateral pressure coefficient is less than 1.0.

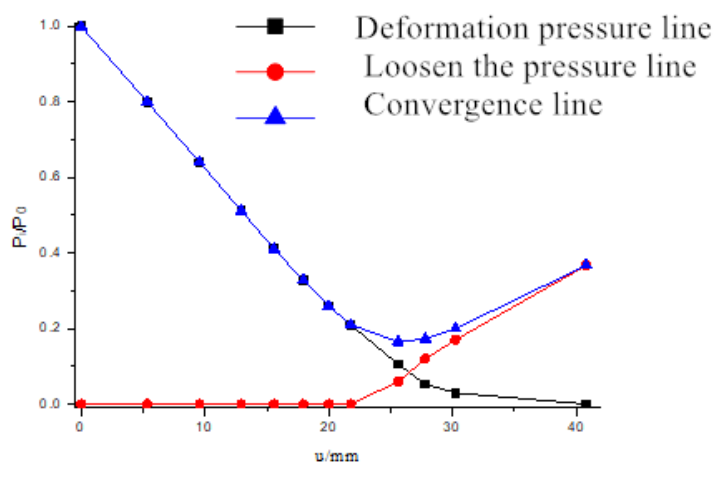

a. Vault

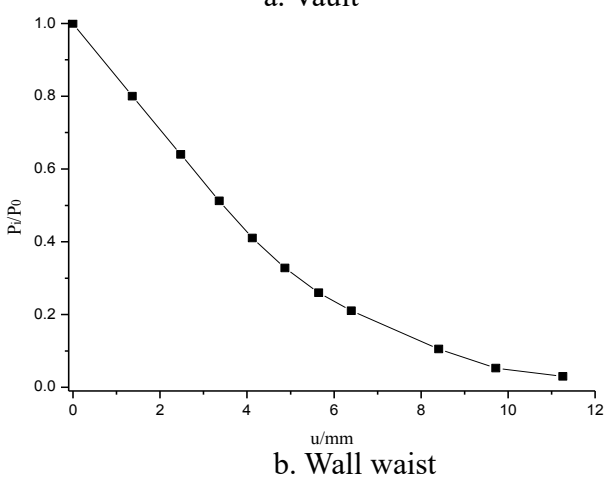

Fig. 5 GRC of tunnel under rock grade V

Due to the expansion of the plastic zone of the 
surrounding rock and the continuous growth of plastic deformation, the slope of the characteristic curve of the surrounding rock gradually turns from negative to positive. At the top of the arch, due to the influence of the loose pressure, the characteristic curve of the surrounding rock has the lowest point. The pressure of the surrounding rock before this point decreases with the increase of the displacement around the cave, and the pressure of the surrounding rock after this point increases with the increase of the displacement around the cave. The elastoplastic boundary point of the characteristic curve of the surrounding rock at the top arch is about $80 \%$ of the initial stress release. The characteristic curve of the surrounding rock at the waist of the wall is composed of a straight line segment and a descending segment curve, the elastoplastic demarcation point is about $40 \%$ of the initial stress release. Like the grade IV surrounding rock, the maximum deformation of the wall waist is less than the maximum deformation of the arch top.

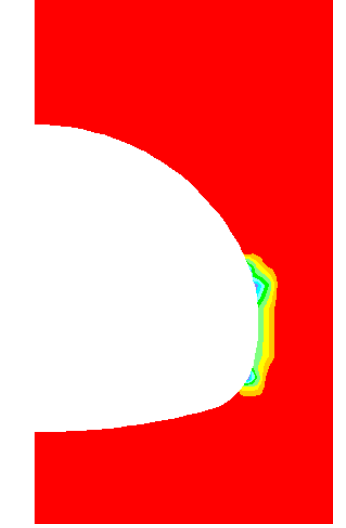

a. $60 \%$ release

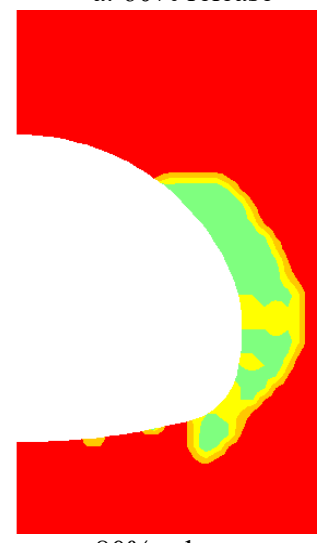

c. $80 \%$ release

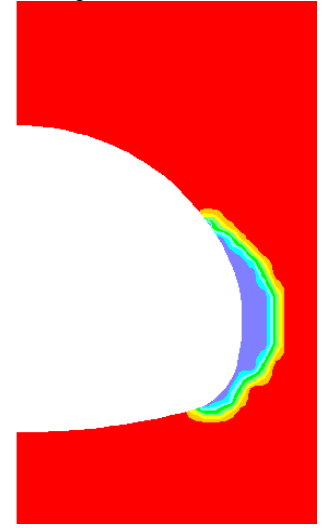

b. $70 \%$ release

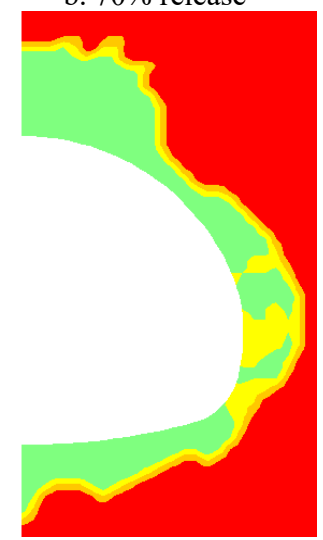

d. $100 \%$ release
Fig. 6 Three lane tunnel plastic zone development process under stress release

\subsection{Two-lane V-level surrounding rock tunnel}

When the size of the tunnel changes from three lanes to two lanes, the height-span ratio of the tunnel changes from 0.68 to 0.755 . As the size of the tunnel changes, the characteristic curve of the surrounding rock also has a certain impact. The maximum deformation at the top of the arch, the two-lane is $30 \%$ lower than the three-lane, the maximum deformation of the wall waist is not obvious. It can be seen from the development of the plastic zone diagram in Fig. 8 that the plastic zone first appeared at the waist of the wall, and then the plastic zone developed to the vault and bottom. The deformation of the surrounding rock cave is still larger than the deformation at the top of the arch than at the waist of the wall.

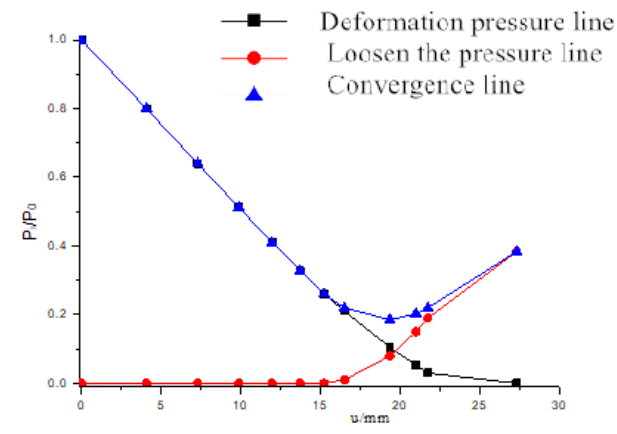

a. Vault

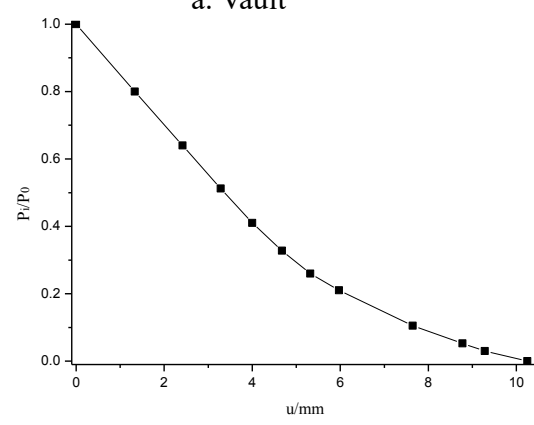

b. Wall waist

Fig. 7 GRC of two lane tunnel under rock grade V

The overall shape of the characteristic curve of surrounding rock at the waist of the wall does not change much, the elastoplastic demarcation point is about $50 \%$ of the initial stress release, and the characteristic curve of surrounding rock has little change compared with three lanes.

The initial stress and surrounding rock parameters of the second and third lanes have not changed significantly, and the lateral pressure coefficient is still less than 1.0. The plastic zone of the surrounding rock appears first at the wall waist. With the further release of the initial stress, the plastic zone develops to the vault and the bottom. In the case of two lanes, the area of the surrounding rock plastic zone is about $10 \%$ smaller than that of a three-lane tunnel.

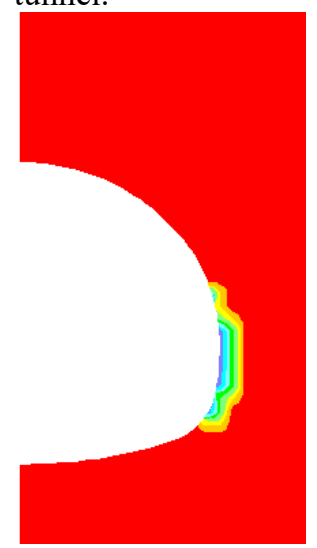

a. $60 \%$ release

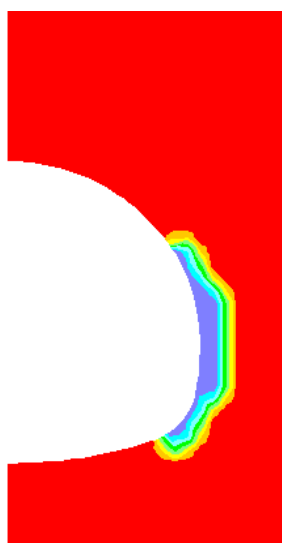

b. $70 \%$ release 


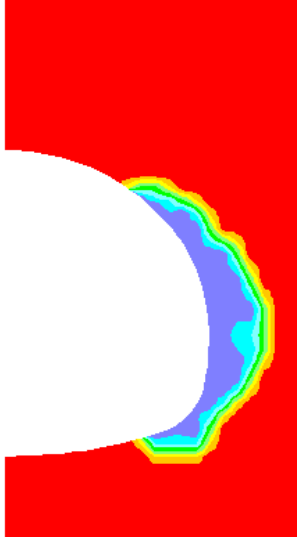

c. $80 \%$ release

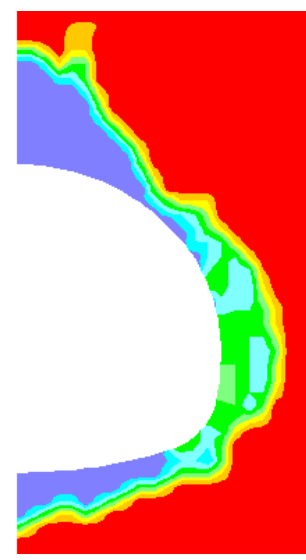

d. $100 \%$ release
Fig. 8 Two lane tunnel plastic zone development process under stress release

\section{Several suggestions for construction and optimization of supporting structure of large-span highway tunnel}

\subsection{Determine the complete support characteristic curve}

When applying the convergence constraint method, the radial displacement that has been generated after the initial support of the front wall of the second liner is a main factor that restricts the application of the convergence constraint method.

Before the second liner support is set up, the radial displacement of the cave wall before the support setting can be determined according to the LDP line, and the construction of the LDP line can be obtained by three-dimensional numerical analysis based on the inversion value without field measurement data.

\subsection{Considerations about the setting of the second lining}

1. In order to avoid being affected by the detonation/shock wave during the blasting operation of the tunnel face, the second liner should be placed far away from the working surface $>(1.5 \sim 2.0) \mathrm{D}$ distance, $\mathrm{D}$ is the hole diameter; The condition of the rock mass is determined by calculation or experience.

2. For most loose and weak surrounding rocks of grade $\mathrm{V}$ and above, when analysis and calculation are required based on the rheological effect of the surrounding rock, the ground stress release and convergence characteristic curve of the surrounding rock can be used, and the construction time of the second lining should make it support The stiffness is within an acceptable range of application.

\section{Conclusion}

According to different lane highway tunnels and surrounding rock levels, this paper obtains the corresponding surrounding rock characteristic curves and surrounding rock plastic zone morphology, and puts forward several suggestions for the construction and optimization of the supporting structure of the arched large-span highway tunnel. The relevant results can be obtained. Provide a certain reference significance for related similar projects. The main conclusions obtained are as follows:

1、In a three-lane tunnel, under the conditions of different levels of surrounding rock, the shape of the convergence characteristic curve of the surrounding rock is not much different, and the displacement value of the tunnel wall and the range of the plastic zone increase significantly with the deterioration of the surrounding rock; The pressure of the surrounding rock corresponding to the lowest point of the convergence characteristic curve of the arch top position increases with the deterioration of the surrounding rock.

2、When the tunnel size changes from three lanes to two lanes, the height-span ratio of the tunnel becomes larger, the maximum deformation at the top of the two-lane arch is $30 \%$ lower than that of the three-lane, and the maximum deformation of the wall waist does not change significantly.

3. As the size of the tunnel changes, the characteristic curve of surrounding rock also has a certain influence.

4. When the initial stress and lateral pressure coefficient of the surrounding rock is less than 1.0, the plastic zone of the surrounding rock of the second and third lane tunnels first appears at the wall waist, and gradually develops to the vault and bottom as the initial stress is further released; After the excavation of the three-lane tunnel is completed, the depth of the plastic zone at the top arch reaches $4 \mathrm{~m}$; in the two-lane tunnel, the plastic zone area of the surrounding rock is about $10 \%$ smaller than that of the three-lane tunnel.

\section{Acknowledgment}

This research was financially supported by the Projects funded by Jiangxi Youth Science Foundation (20181BAB216028), Science and technology project of Jiangxi Provincial Transportation Department (2018H0042), Science and technology project of Jiangxi Provincial Transportation Department(2016H0025).

\section{References}

1. Zhang Lianggang, Research on Deformation Characteristics and Control Technology of Surrounding Rock of Extra-large Fault Slab Tunnel[D]. China University of Geosciences, Beijing: 2014.

2. Hu Shimin. Research on the safety of initial support of subway section tunnels based on the convergence-constraint method[J]. Journal of the China Railway Society, 2015, 37(10): 117-121.

3. Dong He. Research on stability of surrounding rock and supporting structure of highway tunnel[J]. Highway Tunnel, 2016(1): 15-18.

4. Geng Xiaojie. Evaluation method and application of 
tunnel stability under deep buried conditions [D]. University of Science and Technology Beijing, Beijing: 2015.

5. Su Yonghua, Liu Shaofeng, Wang Kaixuan, et al. Stability analysis of underground structures based on convergence-constraint principle[J]. Chinese Journal of Geotechnical Engineering, 2014, 36(11): 2002-2009.

6. Zhang Panfeng, Su Yonghua, Wang Kaixuan, etc. Approximate construction method of stratum characteristic curve for non-circular underground caverns[J]. Hydrogeology and Engineering Geology, 2015, 42(2): 52-58.

7. Zhang Changguang, Fan Wen, Zhao Junhai. New solution and parameter analysis of the plastic zone displacement and characteristic curve of the surrounding rock of deep-buried circular roadway $[\mathrm{J}]$. Rock and Soil Mechanics, 2016, 37(1): 12-24, 32. 